\title{
Uso de aplicativos móviles en el aula y sus factores determinantes
}

\author{
Juan C. Morales ${ }^{1 \star}$, Nicolás E. Ramírez ${ }^{2}$, Steven H. Vargas ${ }^{2}$ y Alfonso J. Peñuela ${ }^{2}$ \\ (1) Decanatura de Innovación y Desarrollo Digital, Universidad Sergio Arboleda, Bogotá, Colombia. \\ (correo-e: juan.morales@usa.edu.co) \\ (2) Escuela de Ciencias Exactas e Ingeniería, Universidad Sergio Arboleda, Bogotá, Colombia. \\ (correo-e: nicolas_ramirez@hotmail.es; stevenh.vargas@correo.usa.edu.co; alfonso.penuela@usa.edu.co) \\ * Autor a quien debe ser dirigida la correspondencia.
}

Recibido Dic. 30, 2019; Aceptado Feb. 29, 2020; Versión final Abr. 13, 2020, Publicado Dic. 2020

\begin{abstract}
Resumen
En este trabajo se busca determinar cómo está siendo percibido el uso de aplicaciones móviles en el aula y qué características influyen para que el docente las use o las descarte. La metodología utiliza un enfoque cuantitativo y se realizó mediante dos encuestas a 131 docentes y 247 estudiantes de la Universidad Sergio Arboleda (Colombia) en el año 2017. Para su evaluación se usó un ANOVA y un modelo Logig binomial. Los resultados muestran que el uso de aplicativos móviles en las aulas de clase no depende de la edad, el género ni la disciplina, aunque hay una mayor tendencia a ser usadas por mujeres jóvenes en ingenierías. El estudio permite concluir que los aplicativos móviles son percibidos como herramientas de apoyo a una necesidad de motivación dentro del aula y su uso por parte del docente depende del tipo de vinculación laboral y de su percepción sobre su importancia.
\end{abstract}

Palabras clave: aplicativos móviles; enseñanza; factores determinantes; percepción; educación superior

\section{Use of Mobile apps in the classroom and its determining factors}

\begin{abstract}
The present study seeks to determine how mobile applications are perceived in the classroom and what characteristics influence professor's app use or rejection. This is a quantitative study and 131 professors and 247 students were surveyed at the Sergio Arboleda University (Colombia) in the year 2017. An ANOVA and a binomial Logig model were used to analyze the data. The results show that the use of mobile applications in the classrooms is not dependent on age, gender, or degree study, although there is a greater tendency by young women in engineering degrees to use mobile apps. It is concluded that mobile apps are perceived as tools for motivation needs within the classroom and that mobile app use by professors depends on the professor's job contract type with the university and on the professor's perception of mobile apps importance.
\end{abstract}

Keywords: apps; teaching; determining factors; perception; higher education 


\section{INTRODUCCIÓN}

El avance acelerado de las tecnologías de la información y la comunicación (TIC) ha influido en la forma en que se enseña en el aula de clase, apoyada en la premisa de que al implementarla, se mejora el proceso de enseñanza-aprendizaje (Sangrà y González-Sanmamed, 2010; Mahenge y Sanga, 2016). Sin embargo, existen importantes dudas sobre la contribución real de las TIC sobre el aprendizaje y sobre el papel que debe jugar, bien como apoyo a pedagogías tradicionales o como una forma radicalmente diferente de enseñar (Livingstone, 2012). Para comprender el rol que juegan las TIC en el proceso de enseñanza-aprendizaje, antes es importante conocer cuáles son las dificultades detectadas y cómo intervienen las TIC en su solución (Morales P. et al., 2018). En este sentido, vemos que el proceso de aprendizaje de un estudiante se ve influenciado por una gran variedad de factores familiares (Kissi, et al., 2018), escolares (Ruíz, 2001) y sociales (Navío-Marco y Solórzano-García, 2019), que afectan el rendimiento estudiantil y determinan el éxito o fracaso en el proceso de aprendizaje del estudiante (Jadue, 2002). También existen factores socio-ambientales que afectan negativa o positivamente en áreas específicas tales como: el lenguaje, la escritura o el cálculo (Sabina et al., 2010).

El rendimiento de los estudiantes también puede verse afectado por factores psicológicos, cuando el estudiante presenta problemas emocionales, manifestándose en: depresión, hiperactividad (Rodríguez 2010), falta de atención, memoria, ansiedad, desórdenes de exteriorización (Jadue, 2002), baja autoestima y en la aceptación de sí mismo (Pérez et al., 2013). El nivel económico y el entorno social, también influyen en la motivación y el rendimiento académico de los estudiantes tal como lo han demostrado diversos estudios (Guisasola et al., 2010; Bogoya et al., 2017).

Para sintetizar el accionar de diversas variables sobre las actitudes de los estudiantes Morales et al., (2018) establecieron y analizaron 4 factores que influyen de manera directa o indirecta sobre el proceso de aprendizaje del estudiante. De esta forma, los autores afirman que las capacidades intelectuales, los vacíos en pre-saberes, la motivación y la dedicación, son los que determinan el éxito o fracaso del estudiante durante el proceso de aprendizaje. Estos factores son observables por el docente con mayor facilidad y a su vez reciben influencia positiva o negativa por otras variables secundarias que en últimas, son las que pueden explicar los comportamientos observados.

En este sentido, afirman los autores que es importante conocer la causa del efecto observable para realizar un manejo adecuado de la dificultad, planteando que las TIC pueden jugar un papel relevante para minimizar estas dificultades. Sin embargo, las TIC por sí solas no parecen solucionar estas dificultades y plantean nuevos retos para su incorporación a la labor docente, como ya lo plantearon en su momento Mishra y Koehler, (2006). En este sentido, Scherer et al., (2017) han determinado que el uso que le dan los estudiantes a las TIC depende del contexto donde se implementen, diferenciando el perfil del usuario en un entorno escolar frente al uso que le dan en un entorno social. Los autores afirman que al reconocer los diferentes patrones de uso de los estudiantes frente a las de las TIC, los docentes podrán incorporarlas de una forma más acertada en su práctica pedagógica. Pero ¿por qué cambia el perfil del estudiante cuando utiliza las los aplicativos móviles en el entorno educativo?

Sobre este particular Sun et al., (2019) plantean que el statu quo del estudiante reduce su motivación para usar los aplicativos móviles en el entorno educativo. En este mismo sentido García-Martín y Cantón-Mayo, (2019) afirman que aunque el uso de aplicativos móviles en las aulas ha aumentado, sigue existiendo una clara tendencia a usarlas fuera del contexto educativo. Sin embargo, Sun et al., (2019) señalan que en la medida en que los estudiantes perciban que los aplicativos móviles serán útiles en la realización de sus trabajos, tenderán a utilizarlas. En el caso de los docentes, la percepción también parece jugar un papel importante en su decisión de implementarla en el aula. Es así como Meishar-Tal y Ronen, (2017) mostraron en su estudio que cuando el docente percibe que tiene poco dominio de la herramienta, intentará evitar su uso para no sentirse inferior a sus alumnos. Sin embargo, Aljaloud et al., (2019) en su estudio realizado en Arabia Saudita determinaron que cuando el docente tiene una percepción positiva del aplicativo móvil y la utiliza, aumenta su disposición a incorporarla en su labor pedagógica. Surgen entonces dos interrogantes: ¿cómo están percibiendo docentes y estudiantes la incorporación de aplicativos móviles en el aula? y ¿qué tan importante es la percepción del docente sobre su decisión de utilizar aplicativos móviles en el aula? En tal sentido, este estudio busca determinar cómo está siendo percibido el uso de aplicativos móviles en el aula y qué características influyen para que los docentes estés dispuestos a usarlas en sus clases.

\section{METODOLOGÍA}

La metodología utiliza un enfoque cuantitativo que busca tener un mayor conocimiento de las dinámicas que influyen en el uso de los aplicativos móviles en las clases a partir de información primaria. Principalmente se busca caracterizar la muestra, identificar su percepción respecto al uso y utilidad de los aplicativos móviles en la clase y determinar la experiencia que han tenido al respecto. 


\section{Población y muestra}

La investigación se realizó mediante la aplicación de dos encuestas estructuradas a docentes y estudiante de 6 facultades de la Universidad Sergio Arboleda durante el mes de noviembre de 2017 seleccionados a conveniencia. En la Universidad laboran tres tipos de docentes; los docentes de tiempo completo, quienes tienen una exigencia laboral de 40 horas semanales, los docentes de hora cátedra, a quienes se les paga por horas y, por último, están los docentes de medio tiempo, quienes tiene una exigencia de 20 horas por semana. En total la muestra quedó conformada por 131 docentes y 247 estudiantes de una población de 3124 estudiantes, 800 docentes de hora catedra, 20 docente de medio tiempo y 253 docentes de tiempo completo para los programas que participaron.

\section{Variables del estudio e instrumentos}

Para el caso de los docentes el instrumento aplicado recogió información de: caracterización de la muestra (género, edad, tipo de docente, semestre a los que dicta clase, facultad a la que pertenece), experiencia en el uso de aplicativos móviles en las clases y dificultad en su adaptación, percepción acerca de su utilidad y criterios que considera más importantes a la hora de implementar dicha tecnología. En la Tabla 1 se muestran los datos obtenidos para algunas variables relevantes que caracterizan a los docentes. Para el caso de los estudiantes las variables analizadas fueron: caracterización de la muestra (género, edad, promedio académico, semestre que cursa, facultad a la que pertenece), clases donde hayan usado aplicativos móviles y dificultad en su adaptación, percepción acerca de su utilidad y criterios que considera más importantes a la hora de implementar dicha tecnología. En la Tabla 2 se muestran los datos obtenidos para las principales variables. Al contrastar los resultados obtenidos para docentes y estudiantes sobre muestras equivalentes, observamos que hay una correlación directa entre las respuestas de ambos grupos. Esto nos asegura la fiabilidad de la información.

Tabla 1: Frecuencia obtenida para encuesta de docentes

\begin{tabular}{|c|c|c|c|}
\hline \multicolumn{2}{|l|}{ Variables } & Frecuencia & $\%$ \\
\hline \multirow{2}{*}{ Género } & Hombre & 94 & 71.8 \\
\hline & Mujer & 37 & 28.2 \\
\hline \multirow{3}{*}{ Tipo de docente } & Docentes de Hora Cátedra & 70 & 53.5 \\
\hline & Docentes de Medio Tiempo & 5 & 3.8 \\
\hline & Docentes de Tiempo Completo & 56 & 42.7 \\
\hline \multirow{6}{*}{ Escuela a la que pertenece } & Escuela de Artes y Música & 3 & 2.3 \\
\hline & Escuela de Ciencias Exactas e Ingeniería & 52 & 39.6 \\
\hline & Escuela de Comunicación & 22 & 16.8 \\
\hline & Escuela de Filosofía y Humanidades & 14 & 10.7 \\
\hline & Escuela de Ciencias de la Salud y el Deporte & 6 & 4.6 \\
\hline & Escuela Internacional de Administración y Marketing & 34 & 26.0 \\
\hline \multirow{6}{*}{ Edad por rangos } & Menos de 27 & 9 & 6.9 \\
\hline & $27-35$ & 42 & 32.0 \\
\hline & $36-44$ & 35 & 26.7 \\
\hline & $45-53$ & 24 & 18.3 \\
\hline & $54-62$ & 16 & 12.2 \\
\hline & Más de 63 & 5 & 3.9 \\
\hline \multirow{2}{*}{$\begin{array}{l}\text { ¿Ha utilizado aplicativos } \\
\text { móviles en el aula? }\end{array}$} & No & 72 & 55.0 \\
\hline & Sí & 59 & 45.0 \\
\hline \multirow{4}{*}{$\begin{array}{l}\text { ¿Considera usted que el uso } \\
\text { de aplicaciones móviles en la } \\
\text { clase le ayudaría en su } \\
\text { proceso de enseñanza? }\end{array}$} & Sí & 96 & 73.3 \\
\hline & No & 10 & 7.6 \\
\hline & No lo ha considerado & 18 & 13.8 \\
\hline & No contesta & 7 & 5.3 \\
\hline
\end{tabular}


Tabla 2: Frecuencia obtenida para encuesta de estudiantes

\begin{tabular}{|c|c|c|c|}
\hline \multicolumn{2}{|l|}{ Variables } & Frecuencia & $\%$ \\
\hline \multirow{2}{*}{ Género } & Hombre & 123 & 49.8 \\
\hline & Mujer & 124 & 50.2 \\
\hline \multirow{5}{*}{ Promedio académico } & De 3,0 a 3,3 & 7 & 2.9 \\
\hline & De 3,4 a 3,6 & 34 & 13.8 \\
\hline & De 3,7 a 3,9 & 81 & 32.7 \\
\hline & De 4,0 a 4,2 & 82 & 33.1 \\
\hline & $\operatorname{De} 4,3$ a 4,6 & 43 & 17.5 \\
\hline \multirow{7}{*}{ Escuela a la que pertenece } & Escuela de Artes y Música & 31 & 12.6 \\
\hline & Escuela de Ciencias Exactas e Ingeniería & 112 & 45.4 \\
\hline & Escuela de Comunicación & 29 & 11.7 \\
\hline & Escuela de Filosofía y Humanidades & 10 & 4.0 \\
\hline & Escuela de Economía & 15 & 6.1 \\
\hline & Escuela de Ciencias de la Salud y el Deporte & 20 & 8.1 \\
\hline & Escuela Internacional de Administración y Marketing & 30 & 12.1 \\
\hline \multirow{6}{*}{ Edad por rangos } & $17-18$ & 42 & 17.1 \\
\hline & $19-20$ & 88 & 35.6 \\
\hline & $21-22$ & 63 & 25.5 \\
\hline & $23-25$ & 39 & 15.7 \\
\hline & $26-30$ & 7 & 2.9 \\
\hline & Más de 30 años & 8 & 3.2 \\
\hline \multirow{2}{*}{$\begin{array}{l}\text { ¿Ha tenido asignaturas donde el } \\
\text { docente haya utilizado aplicativos? }\end{array}$} & No & 119 & 48.2 \\
\hline & Sí & 128 & 51.8 \\
\hline \multirow{4}{*}{$\begin{array}{l}\text { ¿Considera usted que el uso de } \\
\text { aplicaciones móviles en la clase le } \\
\text { ayudaría en su proceso de } \\
\text { aprendizaje? }\end{array}$} & Sí & 197 & 79.8 \\
\hline & No & 9 & 3.6 \\
\hline & No lo ha considerado & 41 & 16.6 \\
\hline & No contesta & 0 & 0.0 \\
\hline
\end{tabular}

\section{Procedimiento y análisis de datos}

Para determinar si existían diferencias significativas entre las variables de estudio, se aplicó un análisis de varianza ANOVA, utilizando el Test de Levene para verificar la homogeneidad de las varianzas y la prueba post hoc de Scheffe. Luego se plantea y desarrolla un modelo Logit binomial con la base de datos de docentes para estimar la probabilidad de que un docente use o no aplicativos móviles en el aula de clase, usando el software estadístico RStudio. Para los cálculos de estadística descriptiva se utilizó el Sofware IBM SPSS Statistics versión 24. Para realizar la estimación del modelo Logit con el fin de determinar la probabilidad que tiene un docente de aplicar dispositivos móviles en el aula se recodificaron las variables tal como se muestran en la Tabla 3.

Tabla 3: Variables del Modelo Logit

\begin{tabular}{|l|l|}
\hline Variable & Descripción \\
\hline $\mathrm{P}(1)$ & Probabilidad de que el docente use aplicativos móviles en el aula. \\
\hline $\mathrm{P}(0)$ & Probabilidad de que el docente no use aplicativos móviles en el aula. \\
\hline TD & Tipo de docente (Incluye dos categorías a saber, 1 si es de tiempo completo y 0 si no lo es) \\
\hline SP & Semestre promedio en el cual imparte asignaturas. \\
\hline EDAD & Edad del Docente. \\
\hline GEN & Género (1 Hombre, 0 Mujer) \\
\hline CUANT & Carreras con alto componente cuantitativo (1 Mayor componente, 0 menor componente). \\
\hline PERC & $\begin{array}{l}\text { Percepción de utilidad (Sí, No, No lo ha considerado) Se recodificó en tres variables dummy: PERCN = No } \\
\text { lo considera útil, PERCNC = No lo ha considerado, PERCS= Sí lo considera útil (línea base) }\end{array}$ \\
\hline ING & Exactas e Ingenierías (0 si pertenece a dicha escuela 1 si no pertenece). \\
\hline
\end{tabular}


Recordemos que dicho modelo busca estimar los parámetros de una relación log lineal de la razón de probabilidades contra la combinación lineal de las variables explicativas, obteniendo así el siguiente modelo luego de la estimación:

$$
\ln \left[\frac{P(1)}{P(0)}\right]=X^{\prime} \hat{\beta}
$$

Siendo $X^{\prime}$ y $\beta$ la matriz conformada por las variables descritas anteriormente y el vector de parámetros estimados vía máxima verosimitud.

\section{RESULTADOS Y DISCUSIÓN}

Los resultados se muestran siguiendo el orden que se planteó en la metodología. En las dos primeras secciones se comentan los hallazgos destacados del análisis estadístico y del ANOVA tanto para los docentes como para los estudiantes. En la tercera sección se muestran los resultados del modelo Logit y se incluyen algunos casos que ayudan a comprender la estimación que realiza el modelo.

\section{Resultados obtenidos: docentes}

A partir del análisis de los datos se logró determinar que el 57\% de los docentes con edades entre 27 a 35 años utilizan aplicativos móviles en sus clases, siendo el grupo que más incursiona en su uso. Sin embargo, no existen diferencias estadísticamente significativas respecto al resto de los grupos. Aunque las mujeres tienden a usar más y con mayor facilidad los aplicativos móviles, se verificó que el género no representa una variable estadísticamente significativa al momento de explicar su uso en el aula, confirmando los resultados obtenidos por Guillén-Gámez et al., (2019). Se evidencio que el $53.6 \%$ de los docentes de tiempo completo y el $40 \%$ de hora cátedra, hacen uso de los aplicativos móviles en su metodología, mientras que de los profesores de medio tiempo, sólo hacen uso un 20\%. Esto podría quedar explicado por el mayor tiempo de que disponen los docentes de tiempo completo para preparar sus clases. Por otra parte, se comprobó que la Escuela de Ciencias Exacta e Ingeniería fue la que hacía mayor uso de aplicativos móviles en el aula con un $59.6 \%$, seguida por la Escuela de Ciencia de la Salud y el Deporte $(50 \%)$ y la Escuela de Ciencias de la Comunicación (45\%). Por otra parte, la que menor uso hace de los aplicativos móviles es la Escuela de Filosofía y Humanidades con un $14 \%$. A pesar de las diferencias observadas tanto por tipo de docente como por Escuela, el ANOVA no evidenció diferencias estadísticamente significativas entre los grupos. Estos resultados son consistentes con los obtenidos por Hrtoňová et al., (2015).

En cuanto a la percepción de los docentes que no han utilizado aplicativos móviles al interior de su clase es de destacar que el $23.6 \%$ señalan que no han considerado su importancia en el proceso de enseñanza, en tanto que el 76.4 no considera que estas herramientas aporten al proceso de enseñanza. Para reducir esta problemática, Marín et al., (2017) señalan la necesidad de diseñar procesos formativos que orienten al docente en cómo incorporar estas herramientas en la clase. Asimismo, se consultó también por los criterios que tiene en cuenta el docente para utilizar un aplicativo móvil en su clase (ver Tabla 4). Basado en lo anterior, se constató que el $64.8 \%$ de los docentes, hacen uso de los aplicativos móviles en el aula con el fin de innovar su estilo de enseñanza, motivar la participación y el interés de los estudiantes y captar con mayor facilidad la atención del estudiante. Por otra parte, el top 4 de aplicativos móviles más usados por los docentes de la Universidad Sergio Arboleda son Kahoot con un 71\%, seguida de aplicaciones como: Google (Drive, Classroom) (15\%), Socrative (12\%) y Geogebra (2\%).

Tabla 4: Criterios valorados por los docentes para usar aplicativos en sus clases

\begin{tabular}{|l|l|l|}
\hline Criterio utilizado & Frecuencia & $\%$ \\
\hline Tener realimentación con los estudiantes & 7 & 5.6 \\
\hline Facilitar la comunicación entre los estudiantes & 11 & 8.8 \\
\hline Hacer seguimiento a los avances de los estudiantes & 17 & 13.6 \\
\hline Innovar el estilo de enseñanza & 39 & 31.2 \\
\hline Captar la atención del estudiante & 19 & 15.2 \\
\hline Centralizar el alojamiento de la información & 3 & 2.4 \\
\hline Seguimiento de la clase en tiempo real & 4 & 3.2 \\
\hline Evaluación en línea & 1 & 0.8 \\
\hline Comunicar a los estudiantes sus calificaciones & 1 & 0.8 \\
\hline Recibir sugerencias en forma anónima & 0 & 0.0 \\
\hline Motivar la participación y el interés del estudiante & 23 & 18.4 \\
\hline
\end{tabular}




\section{Resultados obtenidos: estudiantes}

A partir del análisis de los datos, se logró determinar que los estudiantes más jóvenes ( $<22$ años) son los que han tenido mayor experiencia en el uso de aplicativos en sus clases, aunque no existen diferencias significativas respecto al resto de los grupos. Adicionalmente se determinó que los estudiantes no tienen dificultad relevante para adaptarse al uso de aplicativos móviles y que el género no representa una variable estadísticamente relevante al momento de explicar el uso de aplicaciones móviles en los estudiantes, aunque las mujeres tienden a usarlas en mayor medida y con mayor facilidad. De igual forma, se evidenció que la escuela que, hacia mayor uso de los aplicativos móviles en del aula, es la Escuela de Ciencias Exacta e Ingeniería con un uso del 69.6\%, seguida por la Escuela de Ciencias de la Comunicación (62\%) y la Escuela de Ciencia de la Salud y el Deporte (45\%). Por otra parte, la que menor uso hace de los aplicativos móviles es la Escuela de Filosofía y Humanidades con un $20 \%$. Estos resultados son equivalentes a los obtenidos en la encuesta a los docentes y permiten validar la calidad de la información.

También se recogió información acerca de la percepción de los estudiantes respecto al uso que esperan de los aplicativos móviles al interior de sus clases. Los criterios presentados buscaban identificar algunas necesidades del estudiante que esperan ser atendidas. Los criterios de selección se presentan en la Tabla 5. Los resultados muestran que el $48.6 \%$ de los estudiantes ven la importancia del uso de aplicativos móviles dentro del aula de clase para que el docente innove en su estilo de enseñanza y como una herramienta que motiva su participación. Esto es fundamental pues como lo señalan Taat y Francis, (2020) la percepción de utilidad por parte de los estudiantes es un factor clave para su integración en el aula. Cabe resaltar, que el $79.6 \%$ de los estudiantes, considera que los aplicativos móviles pueden ser un apoyo dentro del aula que facilita su proceso de aprendizaje, coincidiendo con los resultados obtenidos por Mahenge y Sanga, (2016). Como lo señalan Scherer, Rohatgi, y Hatlevik, (2017) al reconocer los diferentes patrones en el uso de los aplicativos móviles por parte de los estudiantes, los profesores pueden incorporarlas correctamente en su práctica pedagógica. A partir del estudio realizado se puede destacar que tanto los estudiantes como los docentes son conscientes de que el uso de las TIC en el aula son una herramienta que influye directa y positivamente en la percepción que tiene el estudiante sobre el proceso y por tanto fomenta su motivación, resultado acorde con el planteamiento de estudios recientes (Silveira et al., 2016, 2019).

Tabla 5: Criterios valorados por los estudiantes para justificar el uso de aplicativos en sus clases

\begin{tabular}{|l|l|l|}
\hline Criterio & Frecuencia & $\%$ \\
\hline Que el avance en la explicación vaya a su ritmo & 22 & $8.9 \%$ \\
\hline Que el docente muestre interés por su aprendizaje & 29 & $11.7 \%$ \\
\hline Que el estilo de enseñanza del docente sea innovador & 69 & $27.9 \%$ \\
\hline Que el método de evaluación sea imparcial & 7 & $2.8 \%$ \\
\hline Que fomente la creatividad & 17 & $6.9 \%$ \\
\hline Que haya facilidad para una adecuada realimentación & 31 & $12.6 \%$ \\
\hline Que la clase motive su participación & 51 & $20.7 \%$ \\
\hline Que las calificaciones sean online & 2 & $0.8 \%$ \\
\hline Que las evaluaciones sean online & 7 & $2.8 \%$ \\
\hline Que mantenga un canal de comunicación & 12 & $4.9 \%$ \\
\hline
\end{tabular}

\section{Resultados obtenidos en el modelo Logit}

Teniendo como punto de partida las variables descritas en la metodología que ayudan a explicar la probabilidad de que un docente use o no aplicativos móviles en el aula, se procede a correr el modelo Logit obteniendo el valor de los parámetros mostrados en la Tabla 6.

Tabla 6: Modelo 1

\begin{tabular}{|l|l|l|l|l|}
\hline & Estimación & Error estándar & Valor $Z$ & $\operatorname{Pr}(>|Z|)$ \\
\hline Intercepción & 1.22298 & 1.06494 & 1.148 & 0.2508 \\
\hline TD & 0.74848 & 0.40332 & 1.856 & $0.0635^{\star}$ \\
\hline SP & -0.07598 & 0.08998 & -0.844 & 0.3984 \\
\hline EDAD & -0.02367 & 0.01876 & -1.262 & 0.2069 \\
\hline GEN & 0.07366 & 0.55001 & 0.134 & 0.8934 \\
\hline PERCN & -1.91836 & 0.86204 & -2.225 & $0.0261^{*}$ \\
\hline PERCNC & -2.99157 & 1.07010 & -2.796 & $0.0052^{* *}$ \\
\hline CUANT & -0.14610 & 0.48182 & -0.303 & 0.7617 \\
\hline
\end{tabular}


Una vez estimado el modelo, se procede a hacer la transformación exponencial de la ecuación (1) para poder hallar la razón de las probabilidades que tienen los diferentes profesores de hacer uso o no de las aplicaciones móviles en el aula. Teniendo dicha proporción, y sabiendo que $P(0)+P(1)=1$, se pueden calcular de manera analítica las probabilidades asociadas a cada registro. La Figura 1 muestra cada registro con sus probabilidades asociadas para el modelo 1, incluyendo las variables TD y PERC.

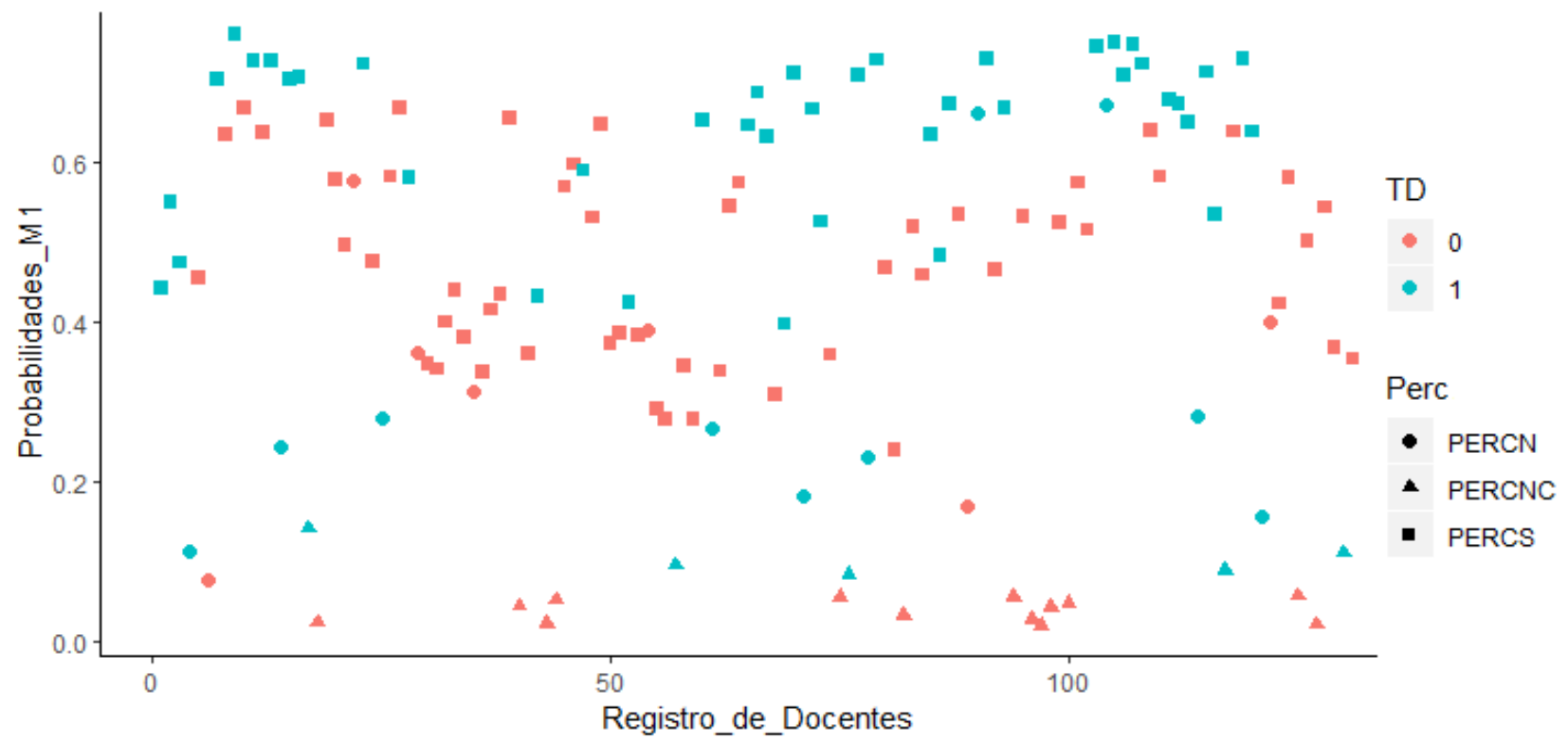

Fig. 1: Registros de las probabilidades de los docentes asociadas para el modelo 1

La Figura 1 muestra una gran dispersión en la estimación de la probabilidad calculada por el modelo 1 para los diferentes individuos. Esto puede deberse a la incorporación de variables no significativas a la hora de explicar la variable dependiente. Las variables que resultaron significativas en el modelo 1 son TD con un efecto positivo y PERC, PERCN con efecto negativo sobre el logaritmo de la razón de probabilidades. Este resultado es muy relevante para este estudio, pues nos indica que el ser profesor de tiempo completo con percepción positiva hacia los aplicativos móviles, aumenta la probabilidad de usarlos en el aula, frente a aquellos profesores que no son de tiempo completo, tal como se observa en la Figura 1. Las otras dos variables indican que una percepción positiva respecto al uso de aplicativos móviles hace que la probabilidad de utilizarlo aumente versus aquellos que no lo consideran importante o no lo han considerado. A partir de los resultados obtenidos se realizó un nuevo modelo con las variables explicativas que resultaron significativas obteniendo los resultados de la Tabla 7.

Tabla 7: Modelo 2

\begin{tabular}{|l|l|l|l|l|}
\hline & Estimación & Error estándar & Valor $Z$ & $\operatorname{Pr}(>|Z|)$ \\
\hline Intercepción & -0.1207 & 0.2553 & -0.473 & 0.6363 \\
\hline TD & 0.7254 & 0.3972 & 1.826 & $0.0678^{*}$ \\
\hline PERCN & -1.8705 & 0.8405 & -2.225 & $0.0261^{*}$ \\
\hline PERCNC & -2.9643 & 1.0520 & -2.818 & $0.0048^{* *}$ \\
\hline
\end{tabular}

En el segundo modelo todas las variables resultan significativas, calculando luego las probabilidades asociadas a cada registro. Como podemos observar en la Figura 2, el modelo logra estimar un conjunto de probabilidades menos dispersa agrupando por rango de probabilidades a los diferentes registros, permitiendo así un perfilamiento de las características del docente a partir del grupo probabilístico en el que se encuentre. A modo de ejemplo, en la Tabla 8 se presentan los cálculos para 4 registros de la muestra con el modelo 2. Como se indicó en cuanto al modelo Logit binomial planteado, se evidenció que el tipo de docente y la percepción son variables significativas que impactan a la probabilidad de uso de los aplicativos móviles en el aula. Este resultado es consistente con los obtenidos por Meishar-Tal y Ronen, (2017) en su estudio y con el estudio de Aljaloud et al., (2019). De acuerdo con los registros, se puede evidenciar que en gran parte se encuentran probabilidades que son acordes con la estimación del modelo 2. Sin embargo, existen algunos casos donde dichas probabilidades no se ajustan de la mejor manera al modelo y estos pocos casos se deben observar con cuidado a la hora de hacer algún tipo de pronóstico. 


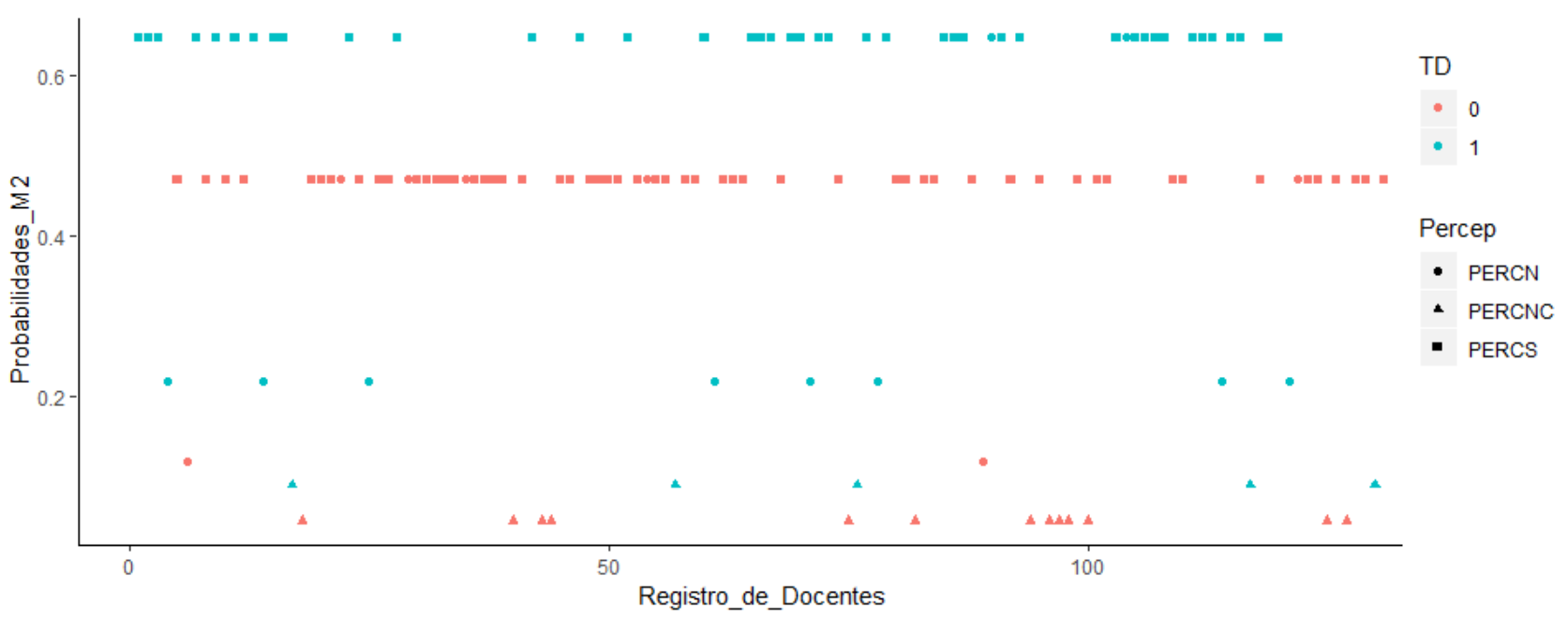

Fig. 2: Registros de las probabilidades asociadas a los docentes para el modelo 2

Tabla 8. Ejemplos de cálculos para 4 registros de la muestra con el modelo 2

\begin{tabular}{|l|l|}
\hline Registro & Detalle \\
\hline Registro número 6: & $\begin{array}{l}\text { Docente que no ha usado aplicativos móviles en el aula, edad 32 años, género femenino, } \\
\text { de carrera con alto componente cuantitativo, docente que no es de tiempo completo, con } \\
\text { percepción positiva. Probabilidad 0.077. }\end{array}$ \\
\hline Registro número 19: & $\begin{array}{l}\text { Docente que sí ha usado aplicativos móviles en el aula, edad 25 años, género masculino, } \\
\text { semestre promedio 3.5, de carrera con alto componente cuantitativo, docente que es de } \\
\text { tiempo completo, con percepción positiva. Probabilidad 0.653. }\end{array}$ \\
\hline Registro número 57: & $\begin{array}{l}\text { Docente que no ha usado aplicativos móviles en el aula, edad 44 años, género masculino, } \\
\text { semestre promedio 4, de carrera con bajo componente cuantitativo, docente que es de } \\
\text { tiempo completo, no ha contemplado la idea. Probabilidad 0.093. }\end{array}$ \\
\hline Registro número 118: & $\begin{array}{l}\text { Docente que sí ha usado aplicativos móviles en el aula, edad 34 años, género masculino, } \\
\text { semestre promedio 2, de carrera con alto componente cuantitativo, docente que no es de } \\
\text { tiempo completo, percepción positiva. Probabilidad 0.638. }\end{array}$ \\
\hline
\end{tabular}

\section{CONCLUSIONES}

De acuerdo con el trabajo presentado y a los resultados obtenidos, se pueden plantear las siguientes conclusiones principales:

1.- Los aplicativos móviles son percibidos como herramientas de apoyo a una necesidad de motivación dentro del aula. No obstante, al mejorar la motivación del estudiante, le facilita su conexión con el proceso.

2.- El uso de aplicativos móviles en las aulas no depende de la edad, el género ni la disciplina, aunque hay una mayor tendencia a ser usadas por mujeres jóvenes en ingenierías.

3.- El uso de aplicativos móviles en el aula depende en gran medida del compromiso y de la percepción que tenga el docente para utilizar estas herramientas como apoyo al proceso de aprendizaje. En tal sentido, si el docente tiene un compromiso con la institución más allá de la clase que dicta y tiene una percepción positiva acerca de la importancia del uso de los aplicativos móviles en el aula, tenderá a hacer uso de estas herramientas como apoyo al proceso de enseñanza.

\section{AGRADECIMIENTOS}

Este trabajo forma parte del proyecto "Incidencia de las TIC en el mejoramiento de las pruebas saber 11: un análisis a partir del modelo TPACK" financiado por el ICFES y la Universidad Sergio Arboleda. Se extiende el agradecimiento a los tres revisores anónimos que ayudaron a mejorar en buena medida el presente artículo.

\section{REFERENCIAS}

Aljaloud, A., Billingsley, W. y Kwan., P., Factors That Influence Teachers' Decisions to Use Smartphone Clicker Apps to Enhance Teacher-Student Interactions in University Classrooms in Saudi Arabia, doi: doi.org/10.1080/23735082.2018.1459802, Learning, 5(1), 67-86 (2019) 
Bogoya, J., Bogoya, J. M. y Peñuela, A., Value-added in Higher Education: Ordinary Least Squares and Quantile Regression for a Colombian Case, doi: doi.org/10.15446/ing.investig.v37n3.61729, Ing. Investig., 37(3), 30-36 (2017)

García-Martín, S. y Cantón-Mayo, I, Use of Technologies and Academic Performance in Adolescent Students, doi: doi.org/10.3916/C59-2019-07, Comunicar, 27(59), 73-81 (2019)

Guillén-Gámez, F., Lugones, A., Mayorga-Fernández, M. y Wang, S., ICT Use by Pre-Service Foreign Languages Teachers According to Gender, Age and Motivation, doi: doi.org/10.1080/2331186X.2019.1574693, Cogent Educ., 6(1), (2019)

Guisasola, J., Almudí, J. y Zuza, K., Dificultades de los Estudiantes Universitarios en el Aprendizaje de la Inducción Electromagnética, doi: doi.org/10.1590/S1806-11172010000100011, Rev. Bras. Ensino Física, 32(1), 1401-9 (2010)

Hrtoňová, N., Kohout, J., Rohlíková, L. y Zounek, J., Factors Influencing Acceptance of E-Learning by Teachers in the Czech Republic, doi: doi.org/10.1016/j.chb.2014.11.018, Comput. Human Behav., 51 (2015)

Jadue, J. G., Factores Psicológicos que Predisponen al Bajo Rendimiento, al Fracaso y a la Deserción Escolar, doi: doi.org/10.4067/S0718-07052002000100012, Estud. pedagógicos, 28, 193-204 (2002)

Kissi, P., Nat, M. y Armah, R., The Effects of Learning-Family Conflict, Perceived Control Over Time and Task-Fit Technology Factors on Urban-Rural High School Students' Acceptance of Video-Based Instruction in Flipped Learning Approach, doi: doi.org/10.1007/s11423-018-9623-9, Educ. Technol. Res. Dev., 66(6), 1547-69 (2018)

Livingstone, S., Critical Reflections on the Benefits of ICT in Education, doi: doi.org/10.1080/03054985.2011.577938, Oxford Rev. Educ., 38(1), 9-24 (2012)

Mahenge, M. y Sanga, C., ICT for E-Learning in Three Higher Education Institutions in Tanzania, doi: doi.org/10.34105/j.kmel.2016.08.013, Knowl. Manag. E-Learning., 8(1), 200-212 (2016)

Marín, F., Inciarte, A., Hernández, H. y Pitre, R., Estrategias de las Instituciones de Educación Superior para la Integración de las Tecnología de la Información y la Comunicación y de la Innovación en los Procesos de Enseñanza. Un Estudio en el Distrito de Barranquilla, Colombia, doi: doi.org/10.4067/S0718-50062017000600004, Form. Univ., 10(6), 29-38 (2017)

Meishar-Tal, H. y Ronen, M., The Impact of Experiencing a Mobile Game on Teachers' Attitudes Towards Mobile Learning, doi: doi.org/10.4018/IJMBL.2017100102, Int. J. Mob. Blended Learn., 9(4), 21-32 (2017)

Mishra, P. y Koehler, M., Technological Pedagogical Content Knowledge: a Framework for Teacher Knowledge, http://doi.org/ 10.1111/j.1467-9620.2006.00684.x, Teach. Coll. Rec., 108(6), 1017-54 (2006)

Morales, J., Ramírez, N. Vargas, S. y Molina, I., Dificultades en el Proceso Enseñanza-Aprendizaje dentro del Aula de Clase e Influencia de las TIC para Minimizarlas; En Las TIC, la Innovación en el Aula y sus Impactos en la Educación Superior por J. C. Morales y S. A. Rodríguez-Jerez, pp. 57-82, Universidad Sergio Arboleda, Bogotá, Colombia (2018)

Navío-Marco, J. y Solórzano-García, M., Student's Social E-Reputation (“karma”) as Motivational Factor in MOOC Learning, doi: doi.org/10.1080/10494820.2019.1579237, Interact. Learn. Environ., in press (2019)

Pérez, M., Valenzuela, M. y otros tres autores, Dificultades de Aprendizaje en Estudiantes Universitarios de Primer Año, doi: doi.org/10.4067/S0718-04622013000200010, Atenea, 508, 135-50 (2013)

Rodríguez, M. C., Factores Personales y Familiares Asociados a los Problemas de Comportamiento en Niños, doi: doi.org/10.1590/S0103-166X2010000400002, Estud. psicol., 27(4), 437-47 (2010)

Ruíz, C., Factores Familiares Vinculados al Bajo Rendimiento, Rev. Complut. Educ., ISSN: 1130-2496,12(1), 81-113 (2001)

Sabina, R., Saéz, B. y Roméu, M., Factores de Riesgo Asociados a Trastornos en el Aprendizaje Escolar: un Problema Sociomédico, MediSur, ISSN: 1727-897X, 8(4), 30-39 (2010)

Sangrà, A. y González-Sanmamed, M., The Role of Information and Communication Technologies in Improving Teaching and Learning Processes in Primary and Secondary Schools, doi: doi.org/10.1080/09687769.2010.529108, Res. Learn. Technol., 18(3), 207-20 (2010)

Scherer, R., Rohatgi, A. y Hatlevik, O, Students' Profiles of ICT Use: Identification, Determinants, and Relations to Achievement in a Computer and Information Literacy Test, doi: doi.org/10.1016/j.chb.2017.01.034, Comput. Human Behav., 70(May), 486-99 (2017)

Silveira, A., Rocha, L., Wildt, C. y Behar, P., Mobile Learning: Pedagogical Strategies for Using Applications in the Classroom, En Proceedings of the 12th International Conference on Mobile Learning, International Association for Development of the Information Society, Vilamoura, Portugal (2016)

Silveira, A., Rocha, L., Wildt, C. y Behar, P., Teaching Practices with Mobile in Different Contexts, doi: doi.org/10.1007/9783-030-02686-8_73, Adv. Intell. Syst. Compu., 880, 982-91 (2019)

Sun, S., Xiong, C. y Chang, V., Acceptance of Information and Communication Technologies in Education: An Investigation into University Students' Intentions to Use Mobile Educational Apps, doi: doi.org/10.4018/IJEIS.2019010102, Int. J. Enterp. Inf. Syst., 15(1), 24-44 (2019)

Taat, M.S., y Francis, A., Factors Influencing the Students' Acceptance of e-Learning at Teacher Education Institute: An Exploratory Study in Malaysia, doi: doi.org/10.5430/ijhe.v9n1p133, Int. J. High. Educ., 9(1), 133-41 (2020) 
